steroids and the addition of chlorambucil permitted withdrawal of steroids. She was treated with chlorambucil for a year and was symptom free on no treatment at the time of reporting. ${ }^{3}$ A review of 15 cases of pulmonary lymphoproliferative conditions including three originally defined as "pseudolymphoma" showed monoclonality in 13 (including all three pseudolymphomas). Patients with diffuse or patchy lung shadowing on radiography had been treated with a mixture of chemotherapy regimens containing steroids, two receiving steroids and chlorambucil alone. One of these was lost to follow up and the other had a clinical response with no change in the radiographic appearance. ${ }^{4}$ Insufficient detail is given in this and other reports ${ }^{8}$ to evaluate the regimens used. Two of a reported series of 13 patients with lymphocytic interstitial pneumonitis were treated with chlorambucil and steroids with improvement in one. ${ }^{10}$ There is no consensus established for the treatment of these conditions. As histologically these patients have low grade lymphoma, we chose to treat our two patients with steroids and chlorambucil, agents well tried and effective for other types of low grade B cell lymphoma and chronic lymphatic leukaemia and used successfully in some patients with "pseudolymphoma". To date this approach has produced a good response in these two patients.

1 Saltzstein SL. Pulmonary malignant lymphomas and pseudolymphomas: classification, therapy and prognosis. Cancer 1963;16:928-55.

2 Gibbs AR, Seal ME. Primary lymphoproliferative conditions of the lung. Thorax 1978;33:140-52.

3 Herbert A, Wright DH, Isaacson PG, Smith JL. Primary malignant lymphoma of the lung. Hum Pathol 1984;15: 415-22.

4 Addis BJ, Hyjek E, Isaacson PG. Primary pulmonary lymphoma: a reappraisal of its histogenesis and its relationship to pseudolymphoma and lymphoid interstitial pneumonia. Histopathology 1988;13:1-17.

5 Li G, Hansmann M-L, Zwingers T, Lennert K. Primary lymphomas of the lung: morphological, immunohistochemical and clinical features. Histopathology 1990;16: 519-31.

6 Robbins R, Peale AR, Al-Saleem T. Pseudolymphomas. AFR 1970;108:149-53.

7 Isaacson PG, Wright DH. Extranodal malignant lymphoma arising from mucosa-associated lymphoid tissue. Cancer 1984;53:2515-24.

8 Kennedy JL, Nathwani NB, Burke JS, Hill RL, Rappaport H. Pulmonary lymphomas and other pulmonary lymphoid lesions. Cancer 1985;56:539-52.

9 Herbert A, Walters MT, Cawley MID, Godrey RC. Lymphocytic interstitial pneumonia identified as lymphoma of mucosa associated lymphoid tissue. $f$ Pathol 1985;146:129-38.

10 Strimlan CV, Rosenow EC, Weiland LH, Brown LR. Lymphocytic interstitial pneumonitis. Review of 13 cases. Ann Intern Med 1978;88:616-21.

11 Goldman JM. The lymphomas. In: Hoffbrand AV, Lewis SM, eds. Postgraduate haematology. 3rd edn. Oxford: Heinemann, 1989:502-29.

12 Krikorian JG, Portlock CS, Cooney DP, Rosenberg SA. Spontaneous regression of non-Hodgkin's lymphoma: a report of nine cases. Cancer 1980;46:2093-9.
Culture of

\section{Mycobacterium kansasii} in the blood of an HIV negative patient

\author{
D Veale, D Fishwick, J E S White, \\ A D Gascoigne, K Gould, P A Corris
}

\begin{abstract}
A 23 year old man with a congenital myelodysplastic disorder and fibrosing lung disease received treatment with prednisolone. After nine months his condition deteriorated and Mycobacterium kansasii was isolated from blood cultures and lymph node biopsy specimens. He responded to antituberculous treatment. $M$ kansasii has not previously been isolated from the blood stream of HIV negative patients.
\end{abstract}

(Thorax 1993;48:672-673)

Mycobacterial bacteraemia with organisms other than $M$ tuberculosis (mycobacteria other than tuberculosis, MOTT) is not unusual in patients with acquired immunodeficiency syndrome (AIDS), ${ }^{1}$ and the $M$ avium complex is the most frequently isolated subtype. ${ }^{2}$ There are few reports of isolation of nontuberculous mycobacteria from blood cultures in non-HIV patients. ${ }^{3}$ We report a young man with a familial myelodysplasia and persistent Epstein-Barr virus infection who developed widespread $M$ kansasii infection with isolation of the organism from blood cultures.

\section{Case report}

A 23 year old man developed fever and rigours five weeks after returning from a holiday in Spain. He then developed a cough with clear sputum and nausea. In his past history he had suffered severe varicella and recurrent herpes simplex infections and a persistent Epstein-Barr virus infection from the age of 20 years. An older brother had died of refractory anaemia with excess lymphoblasts. Genetic studies had determined familial dysplasia with constitutional inversion of chromosome $1 .^{4}$ Immunological studies had shown leucopenia with a profound lymphopenia but no other abnormality. On examination he had gross digital clubbing and generalised lung crackles. White blood cell count (WBC) was $0.9 \times 10^{9} / 1$ and he was HIV antibody negative. Chest radiography showed diffuse patchy parenchymal shadow- 
ing. He was treated with broad spectrum antibiotics but showed no clinical response and was referred to the regional respiratory centre. Open lung biopsy samples showed desquamative pneumonitis typical of Hamman-Rich syndrome. Specimens were cultured and examined on a weekly basis, but there was no evidence of fungi, mycobacteria, or pneumocystis pneumonia after eight weeks. He was treated with high dose steroids and showed an immediate clinical and functional improvement.

Twelve months later, while taking $20 \mathrm{mg}$ prednisolone and acyclovir $200 \mathrm{mg}$ three times a day, he became unwell and had a recurrence of fever. No lymph nodes were palpable but he had persistence of lung crackles. His haemoglobin was $9 \cdot 2 \mathrm{~g} / \mathrm{dl}$ and his WBC $1.4 \times 10^{9} / 1$. One week later repeat chest radiography showed the new development of mediastinal adenopathy and a diagnosis of Epstein-Barr driven lymphoma was suspected. Large fleshly lymph nodes were found at mediastinoscopy, and histological examination revealed numerous acid fast bacilli which proved to be $M$ kansasii on culture. He also developed a large pericardial effusion which required drainage. $\mathrm{He}$ was initially treated with standard antituberculous chemotherapy comprising rifampicin, isoniazid, and pyrazinamide. He deteriorated, however, on this regimen and treatment was therefore changed to rifabutin, isoniazid, ethambutol, and ciprofloxacin with gradual and sustained improvement. $M$ kansasii was isolated from blood cultures, sputum, pericardial fluid, and mediastinal tissue. All specimens were inoculated onto Lowenstein Jensen slopes and tissue specimens into Kirchner's broth. Blood cultures were inoculated directly into the broth, then saponised. The organism was sensitive to rifabutin, rifampicin, ethionamide, ciprofloxacin, capreomycin, ethambutol, and cycloserine but resistant to isoniazid, streptomycin, and pyrazinamide. No synergy tests were performed. He did not suffer any relapse of symptoms following completion of 12 months antituberculous treatment. The dosage of prednisolone was gradually reduced and he has returned to active sports.

\section{Discussion}

$M$ kansasii has been isolated from many environmental sources including tap water ${ }^{5}$ and grows best at $37^{\circ} \mathrm{C}$. It is usually sensitive to rifampicin and ethambutol. ${ }^{6}$ In immunocompetent patients treatment for between three and 24 months resulted in cure in all cases with no relapses after a mean follow up of five years. $M$ kansasii has been recorded in pericardial effusion in one previous report. ${ }^{7}$

Disseminated infection with MOTT was first reported by Koch and Rabinowitsch in $1907 . .^{8}$ There have been numerous reports of disseminated $M$ avium complex infection in patients with $\mathrm{AIDS},{ }^{29}$ but a study of 134 patients who were immunosuppressed from causes other than HIV showed no isolation of MOTT. ${ }^{1}$ Disseminated infection with $M$ kansasii has been reported in patients with AIDS. ${ }^{10}$ Pierce et al isolated MOTT from blood in eight patients with non-HIV related immunodeficiency, all of whom were on long term steroids for a variety of conditions including preleukaemia, ${ }^{3}$ but in none of these was the organism $M$ kansasii.

Bacteraemia with mycobacteria other than $M$ avium complex is a rare event and occurs most commonly in immunosuppressed patients. ${ }^{3}$ Isolation of $M$ kansasii from blood is rare in HIV patients and previously unknown in non-HIV patients. It may be that the organism is seldom present in blood, but it is most probably because blood culture specimens are taken too infrequently. Technical inadequacies may also play their part, but the development of radiometric detection methods may improve the yield of positive results. ${ }^{11} 12$

As MOTT in immunosuppressed patients may well be more responsive to treatment in non-HIV patients ${ }^{13}$ than in those with HIV, it is important to culture specimens including frequently obtained blood samples for a prolonged period with appropriate media in relevant clinical situations.

We wish to thank the Respiratory Pathogens section of the Public Health Laboratory, Newcastle upon Tyne, for their help with the isolation and identification of the mycobacteria.

1 Peters M, Schurmann D, Mayr AC, Hetzer R, Pohle HD, Ruf B. Immunosuppression and mycobacteria other than Mycobacterium tuberculosis; results from patients with and without HIV infection. Epidemiol Infect 1989; 103:293-300.

2 Green JB, Sidhu GS, Lewin S, Levine JF, Masur H, Simberkoff MS, et al. Mycobacterium aviumintracellulare: a cause of disseminated life-threatening infection in homosexuals and drug abusers. Ann Intern Med 1982;97:539.

3 Pierce PF, DeYoung DR, Roberts GD. Mycobacteremia and the new blood culture systems. Ann Intern Med 1983;99:786-9.

4 Paul B, Reid MM, Davison EV, Abela M, Hamilton PJ. Familial myelodysplasia: progressive disease associated with emergence of monosomy 7. Br f Haematol 1987; 65:321-3.

5 Joyson DM. Water; the natural habitat of $M$ kansasii. Tubercle 1979;60:77.

6 Banks J, Hunter AM, Campbell IA, Jenkins PA, Smith PA. Pulmonary infection with $M$ kansasii in Wales, 1970-9; review of treatment and response. Thorax 1983; 38:271.

7 Palmer JA, Watanakunakorn C. Mycobacterium kansasii pericarditis. Thorax 1984;39:876-7.

8 Koch M, Rabinowitsch L. Die tuberkulose der vogel und ihre beziehungen zur sangertiertuberkulose. Virchows Arch Pathol Anat 1907;190:246.

9 Zakowski P, Fligiel S, Berlin OGW, Johnson BL Jr. Disseminated Mycobacterium avium-intracellulare infection in homosexual men dying of acquired immunodeficiency. $\Im A M A$ 1982;248:2980.

10 Sherer R, Sable R, Sonnenberg M, Cooper S, Spencer P, Schwimmer $S$, et al. Disseminated infection with Mycobacterium kansasii in the acquired immunodeficiency syndrome. Ann Intern Med 1986;105:710-2.

11 Salfinger M, Stool ED, Piot D, Heifets L. Comparison of three methods for recovery of Mycobacterium avium complex from blood specimens. $f$ Clin Microbiol 1988; 26:1225-6.

12 Kiehn T, Cammarata $R$. Comparative recoveries of $M y$ cobacterium avium- $M$ intracellulare from isolator lysiscentrifugation and BACTEC $13 \mathrm{~A}$ blood culture systems. F Clin Microbiol 1988;26:760-1.

13 Horsburg CR, Mason UG, Farhi DC, Iseman MD. Disseminated infection with Mycobacterium avium-intracellulare: a report of 13 cases and a review of the literature. Medicine 1985;64:36-48. 